\title{
Role of 18FDG-PET/CT in evaluation of breast cancer metastasis
}

\author{
Medhat M. Reffat ${ }^{a}$, Haney H. Lotfy ${ }^{b}$, Ahmed L. Salem ${ }^{\text {a }}$
}

a Department of Radiology, Benha faculty of medicine, Banha University, Egypt. b Department of Radiology, Almaadi Armed Forces compound, Military Medical Academy , Cairo , Egypt.

Correspondence to: Ahmed L. Salem, Department of Radiology, Benha faculty of medicine, Banha University, Egypt.

Email:

drahmedlabeb1983@gmail.com

Received: 10 July 2021

Accepted:30 November 2021

\begin{abstract}
:
Background: Worldwide breast cancer is the most frequently diagnosed malignancy among women and it is the most common leading cause of cancer death. The main applications of FDGPET/CT in breast cancer are detection of malignancy, re-staging, detection of metastatic disease and for monitoring treatment response. Objective: This prospective study aimed to evaluate the role of $\left({ }^{18}\right.$ FDG- PET/CT $)$ in breast cancer metastasis. Patients and methods: Fifty female patients with breast cancer were included in this study. Results: PET/CT showed high sensitivity and specificity to detect breast cancer metastasis involving loco-regional metastasis (nodal) and distant metastasis. Bone is the most frequent site of distant metastasis in breast cancer patients. Conclusion: PET/CT can detect metastatic spread in breast cancer and monitor therapy response.
\end{abstract}

Keywords: [18] fluorodeoxyglucose-positron emission tomography /computed tomography $\left({ }^{18} \mathrm{FDG}-\mathrm{PET} / \mathrm{CT}\right)$; breast cancer; distant metastasis.

\section{Introduction:}

Worldwide breast cancer is the most frequently diagnosed malignancy among women and it is the most common leading cause of cancer death with progressive increased incidence over the last few decades [1].
Many risk factors are well-known; however, the exact causes of breast cancer have not been identified. Family history of breast cancer, also genetic mutations are considered to convey a very strong risk factors of developing the disease [2]. 
Early detection of breast cancer is considered to be an essential milestone in the control of breast cancer. Early diagnosis is crucial in determining the choice of therapy, as well as a patient's prognosis and chances for survival [3].

$\mathrm{PET} / \mathrm{CT}$ is a new imaging modality in oncology it has re-defined as the gold standard for oncologic imaging compared with other imaging modalities to demonstrate proper extent of the disease and metastatic spread within a single noninvasive investigation [4].

Integrated positron emission tomography (PET) and computed tomography (CT) performed with fluorine $18 \quad\left({ }^{18} \mathrm{~F}\right)$ fluorodeoxyglucose (FDG) is one of the functional imaging modalities used to visualize glucose metabolism in living human tissues. Given its high sensitivity in detection of malignancy, FDG PET/CT is increasingly being used in evaluation of oncology patients [5].

PET/CT collect between the CT anatomical information and PET functional information leading to increase the ability in different types of clinical oncology and provides an effective and accurate imaging technique for a variety of diagnostic oncology. In breast cancer, main applications were detection of the response, restaging, detection of metastatic disease and for monitoring treatment response [6].

PET/CT play a very important role in the diagnosis of locoregional metastasis (including nodal) and distant metastasis in helping to plane surgical and medical treatment as well as monitoring the response to treatment [7].

The aim of this work: The aim of the work is to evaluate the role of FDG PET/CT in evaluation of breast cancer metastasis.

\section{Patients and Methods:}

This is a prospective study in which fifty female patients with evidence of proven metastatic breast cancer were referred for PET/CT in Department of Nuclear Medicine in Egyptian Armed Forces Hospitals in the period between November 2015 and December 2020. The mean age of the studied patients was 57 years, with a standard deviation of 13 years. About twothirds of the patients showed positive family history. Half of the patients $(50.0 \%)$ had right breast cancer, forty-four percent had left breast cancer, and only $6 \%$ had bilateral breast cancer.

The research ethics committee (Faculty of medicine-Benha University) approved the study protocol, and all patients were 
enrolled after written informed consent was obtained. Patients were recruited from Radiodiagnosis, Nuclear medicine departments. The regional ethics committee approved the study and written informed consent were obtained from all participants.

Inclusion criteria were: All patients included in the study with PET/CT on account of evaluation of metastatic breast cancer; these were evident by elevated tumor markers, equivocal findings on CT and MRI.

- Initially metastatic breast cancer or relapsed after primary treatment evident by abdominal ultrasound, CT scan, bone scan or elevated tumor markers.

- FDG-PET/CT studies were performed shortly after appearance of clinical, radiological and/or laboratory data warranting restaging and follow up.

\section{Exclusion criteria:}

- Patients with strong history of hypersensitivity disorders to the CT contrast agents.

- Patients with renal function impairment (with serum creatinine > $2 \mathrm{mg} / \mathrm{dl}$ ) because they are liable to contrast nephropathy.
- Pregnant patients as the patient have high dose of radiation exposure.

- Overweight patients that have difficulties in accessing the scanners and table movement.

\section{Contrast-enhanced CT Protocol:}

Contrast-enhanced CT of the chest, abdomen, and pelvis was performed by using a 64-detectors CT scanner. Patients were given $200-800 \mathrm{~mL}$ of $2 \%$ oral contrast material divided at 45 and 15 minutes before the examination [8].

Intravenous bolus injection of a nonionic iodinated contrast material at a dose of 2$3 \mathrm{~mL} / \mathrm{KG}$ of body weight was performed just before initiation of scanning [8].

Scans were acquired from the thoracic inlet to the pelvic floor by using 2.5 -mm-thick sections, and contiguous 5-mm axial image reconstruction [8].

Scanning protocols with $120 \mathrm{kVp}$ and effective tube current that varied from 60 to $140 \mathrm{mAs}$ were used [8].

\section{PET-CT Protocol:}

Patients fasted for at least 4 hours before the examination, and blood glucose levels were less than $140 \mathrm{mg} / \mathrm{dL}$. A dose of (0.18$0.21 \mathrm{mCi} / \mathrm{kg}$, minimum $3 \mathrm{mCi}$ ) FDG was injected intravenously. The patients rested 
in a quiet room. After the 45-60-minute uptake period, the patients were taken for the PET-CT study [9].

No oral or intravenous contrast agent was used for the CT part of the PET-CT examination.

A section thickness of $4 \mathrm{~mm}$ and a pitch of 1 were used. After CT acquisition, PET acquisition of the same axial range begun with the patient in the same position on the table for 2-3 minutes per bed position PET data were acquired by using a matrix of $128 \times 128$ pixels. CT- based attenuation correction of the emission images was used [9].

After PET data acquisition was completed, the reconstructed attenuation corrected PET images, CT images, and fused images of matching pairs of PET and CT images were available for review in axial, coronal, and sagittal planes, as well as in maximum intensity projections and in three-dimensional cine mode [9].

\section{Results:}

\section{Statistical methods}

Data management and statistical analysis were done using SPSS version 25. (IBM, Armonk, New York, United States). Numerical data were summarized as means and standard deviations or medians and ranges. Categorical data were summarized as numbers and percentages. CT and $\mathrm{PET} / \mathrm{CT}$ were compared regarding detection of metastasis in different sites using the McNemar test. The number of metastasis detected by $\mathrm{CT}$ and PET/CT in different sites was compared using Wilcoxon signed ranks test. All P-values were two-sided. $\mathrm{P}$ values less than 0.05 were considered significant.

\section{Number of metastasis in different sites by} CT and PET/CT (Table 1 \& Figure 1):

\section{- Lymph nodes metastasis}

The median number of lymph node metastasis detected by PET/CT was significantly higher (5) than that of CT (3). P-value was $<0.001$.

\section{- Bone metastasis}

$>$ The median number of bone metastasis detected by PET/CT was significantly higher (12) than that of CT (7). Pvalue was 0.028 .

\section{- Liver metastasis}

The median number of liver metastasis detected by PET/CT was significantly higher (5) than that of CT (2). P-value was 0.015 .

\section{- Loco-regional}

$>$ The median number of locoregional metastasis detected by PET/CT was 
significantly higher (4) than that of CT

(2). P-value was $<0.001$.

\section{- Lung}

There were no significant differences between $\mathrm{CT}$ and $\mathrm{PET} / \mathrm{CT}$ regarding the number of metastasis detected in the lung $(\mathrm{P}$-value $=0.187)$ and suprarenal $(\mathrm{P}$-value $=1.0)$.
- Suprarenal

$>$ There were no significant differences between $\mathrm{CT}$ and PET/CT regarding the number of metastasis detected in the suprarenal $(\mathrm{P}$-value $=1.0)$.

(Table 1): Number of metastasis in different sites by CT and PET/CT.

\begin{tabular}{lcc}
\hline & Median (range) & P value \\
\hline Lymph nodes & $3(1-15)$ & \\
CT & $5(1-42)$ & \\
PET/CT & & \\
Lung & $4(1-52)$ & 0.001 \\
CT & $3(1-38)$ & \\
PET/CT & & \\
Bone & $7(1-89)$ & 0.028 \\
CT & $12(1-59)$ & \\
PET/CT & $2(1-47)$ & \\
Liver & $5(1-30)$ & 0.015 \\
CT & & \\
PET/CT & $1(1-2)$ & 1.0 \\
Suprarenal & $2(1-2)$ & \\
CT & & \\
PET/CT & $2(1-4)$ & $<0.001$ \\
Locoregional & $4(1-9)$ & \\
CT & \\
PET/CT &
\end{tabular}

Wilcoxon signed ranks test was used 


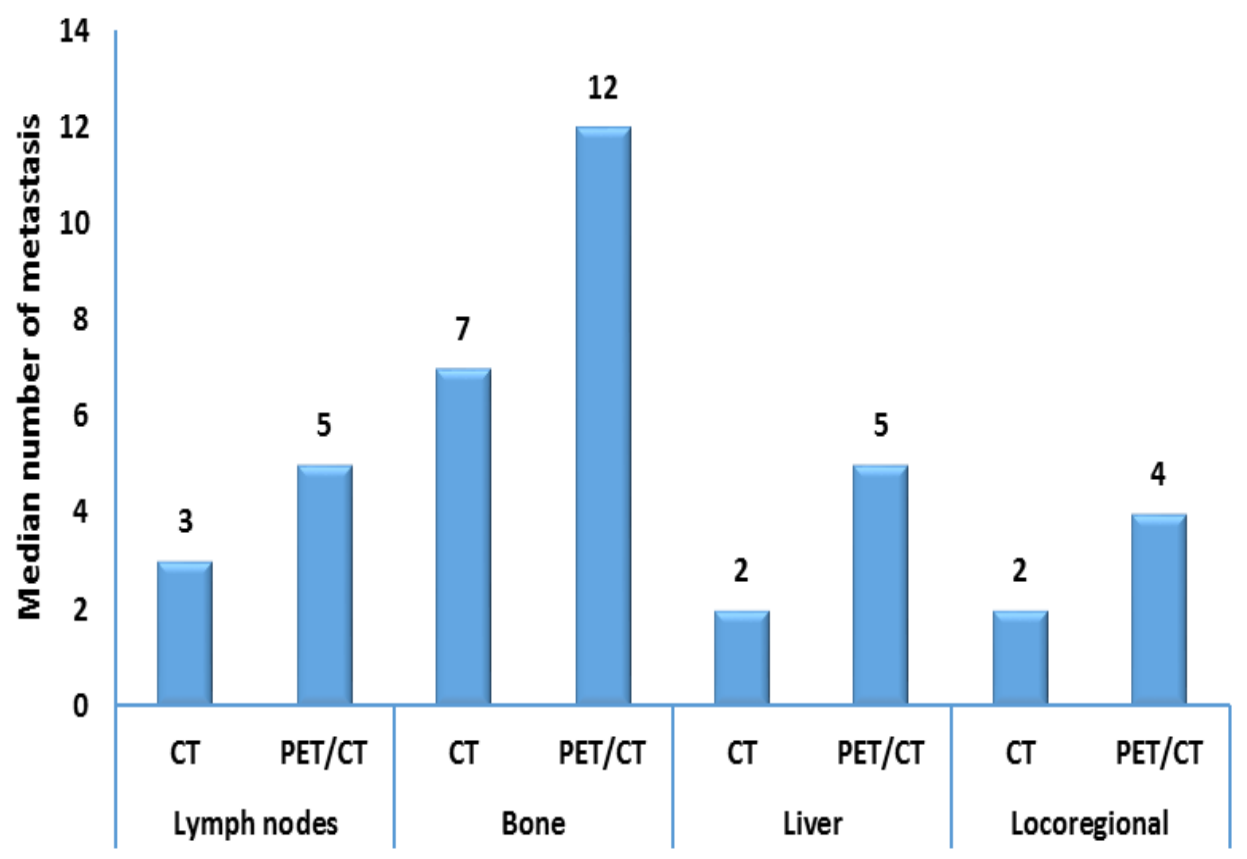

Figure 1: Number of metastasis in different sites by CT and PET/CT.

\section{Discussion:}

Worldwide, breast cancer is the most frequently diagnosed life threatening cancer in women and a leading cause of death [10].

Since breast tumors represent a collection of different histopathological types, understanding metabolic differences between molecular subtypes offers a way to identify new subtype-specific treatment strategies, especially if metabolite changes are evaluated in the broader context of the network of enzymatic reactions and pathways [11].
Imaging techniques play an important role in the detection of metastatic spread in breast cancer, such early detection improves patients' prognosis, and has a substantial influence on therapy management, especially using molecular imaging with PET-CT [12].

Also monitoring treatment responses with PET-CT for women with metastatic breast cancer can be difficult and with the goals of therapy focused on improving quality of life and overall survival, finding a test that is 
safe, non-invasive and reliable to assess response is a challenge [13].

Although tumor size, lymph node metastasis and tumor grade have been widely accepted in daily clinical practice, the identification of further prognostic indicators. Calculation of metabolic SUV max appears to be useful for selecting patients who have inferior prognosis and need further adjuvant treatment of different breast cancer subtypes [14].

In this prospective study which includes 50 female patients with metastatic breast cancer, we try to detect the diagnostic value of PET/CT in metastatic breast cancer patients. PET/CT was requested for initial staging of the included cases then evaluated qualitatively and semi quantitatively with calculation of SUV max.

A retrospective study was done which included 225 patients with breast cancer patients and compared the sensitivity and specificity of PET/CT and conventional imaging (CT, Ultrasonography, radiography, and skeletal scintigraphy) for the detection of distant metastases and they concluded that, in the detection of such metastases, PET/CT was superior to conventional imaging in terms of both sensitivity and specificity [15].
In the present study, using PET/CT showed high sensitivity and specificity to differentiate between metastatic lesions and this result agrees with the previously reported results.

Regarding bone metastasis, bone is the most frequent site of distant involvement in breast cancer. In our study, the median number of bone metastasis detected by PET/CT was significantly higher (12) than that of CT (7). P-value was 0.028 .

Another study was done and in their results Twenty-nine consecutive women with breast cancer were assessed with CT scan, bone scintigraphy and wholebody FDG-PET/CT. The sensitivity of CT scan and bone scintigraphy was $76 \%$ (53/70) compared to $96 \%$ (67/70) for FDG$\mathrm{PET} / \mathrm{CT}$. The specificity of CT scan and bone scintigraphy and FDG-PET/CT was 95\% and $92 \%$, respectively. On a lesionbasis whole-body FDG-PET/CT is more sensitive and equally specific for the detection of bone metastases compared with CT scan and bone scintigraphy [16]. Similarly another reported high sensitivity, specificity and accuracy of $85 \%, 90 \%$ and $88 \%$, respectively for FDG- PET in detection of metastatic spread to lymph nodes in mediastinal and intra-mammary groups. While CT was able to provide 
sensitivity, specificity and accuracy of only $54 \%, 85 \%$ and $73 \%$ in same patients groups [17].

In our study, the median number of lymph node metastasis detected by PET/CT was significantly higher (5) than that of CT (3). $\mathrm{P}$-value was $<0.001$.

Also the median number of loco-regional metastasis detected by PET/CT was significantly higher (4) than that of CT (2). $\mathrm{P}$-value was $<0.001$.

Regarding pulmonary metastasis, diagnostic CT efficiently detected sub-centimetric pulmonary nodules. Whereas in PET imaging in view of partial-volume effect and respiratory movements, PET lacked sensitivity for detecting smaller nodules. Combined PET/CT examination obviously improved the sensitivity of PET/CT in comparison to stand alone PET . There were no significant differences between $\mathrm{CT}$ and PET/CT regarding the number of metastasis detected in the lung $(\mathrm{P}-\mathrm{value}=0.187)$.

FDG PET/CT had sensitivity for liver metastases slightly higher than that of conventional imaging, PET/CT helped to classify doubtful findings on conventional imaging (angiomas and cysts), as reported in another study on 115 breast cancer patients they found that PET/CT is able to assess primary tumor size and metastatic spread more accurately than traditional diagnostic methods. It can detect distant metastases in $7-8 \%$ of those patients who were declared free of metastasis. PET/CT scan modifies the disease stage determined by traditional diagnostic modalities in almost half of the patients and leads to a change in the treatment plan in every 6th patient [18].

In our study, the median number of liver metastasis detected by PET/CT was significantly higher (5) than that of CT (2). P-value was 0.015 .

The FDG uptakes of metastatic sites were evaluated using the SUV max ( $\mathrm{SUV}>2.5$ is considered suspicious for malignancy) [19].

\section{Illustrative cases}

\section{Case No. 1}

A 37 years old female patient with history of left breast cancer since April 2013 underwent surgical lumpectomy at September 2014, followed by multiple cycles of chemotherapy and radiotherapy and kept on hormonal therapy.

Whole body FDG PET/CT was done with multiple metastatic deposits denoted at left sub-pectoral lymph nodes, retrosternal lymph nodes, right hepatic lobe and both iliac bones. 
The following images show the sites of

metastatic deposits (Figure 2, $3 \& 4$ ).

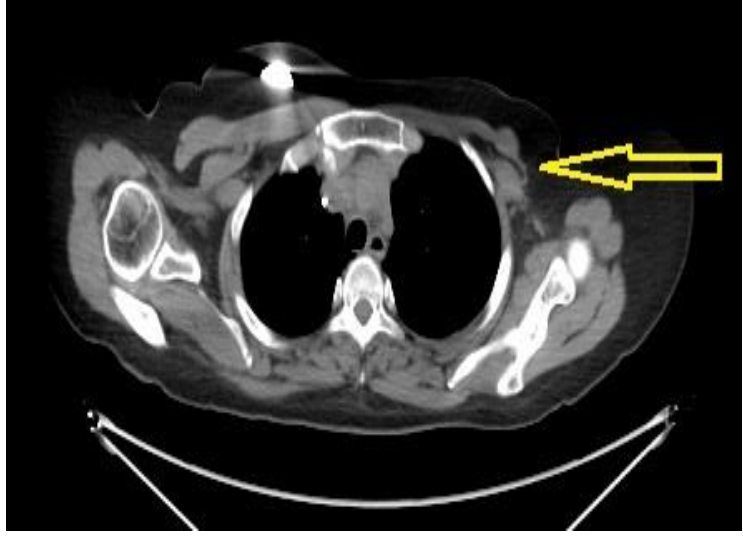

A

Figure (2): a. CT scan of the chest (soft tissue window axial cuts) showed enlarged left sub-pectoral lymph nodes, the largest measure $25 \mathrm{~mm}$ (yellow arrow). b. PET/CT (axial images) showed enlarged FDG-avid left sub-pectoral lymph nodes (yellow arrow), its SUVmax = 7.5, also showed enlarged FDG-avid retro-sternal lymph nodes (blue arrow), its SUVmax $=8$; consistent with lymph nodes metastatic deposits.

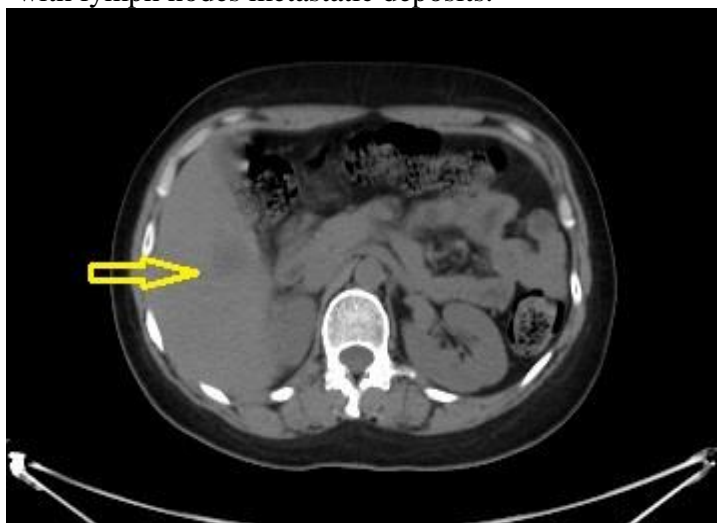

A

Figure (3): a. CT scan of the abdomen (soft tissue window axial cuts) showed ill-defined hypo-dense right hepatic lobe focal lesion seen at sub-segment $\mathrm{V}$ measure about $3.5 \times 3 \mathrm{~cm}$ (yellow arrow). b. PET/CT (axial images) showed FDG avid right hepatic lobe focal lesion seen at sub-segment V with SUVmax $=10.8$ (yellow arrow) consistent with hepatic metastatic deposit.

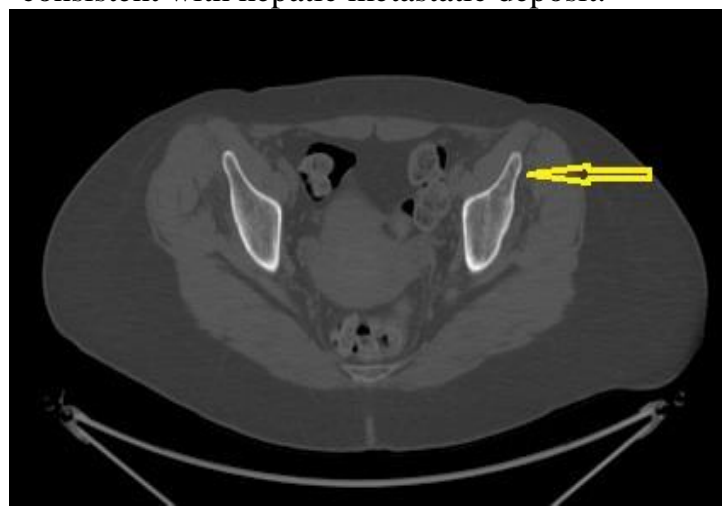

A

Figure (4): a. CT scan of the pelvis (bone window axial cuts) showed an ill-defined intra-osseous sclerotic bony lesion is seen at the left iliac bone (yellow arrow).b. PET/CT (axial images) showed FDG-avid intra-osseous bony lesion is seen at the left iliac bone (yellow arrow) with SUVmax $=5.7$ consistent with metastatic bony deposits. 


\section{Case No. 2}

A 52 years old female patient with history of right breast cancer since October 2015 underwent surgical lumpectomy at January 2016, followed by multiple cycles of chemotherapy and radiotherapy.

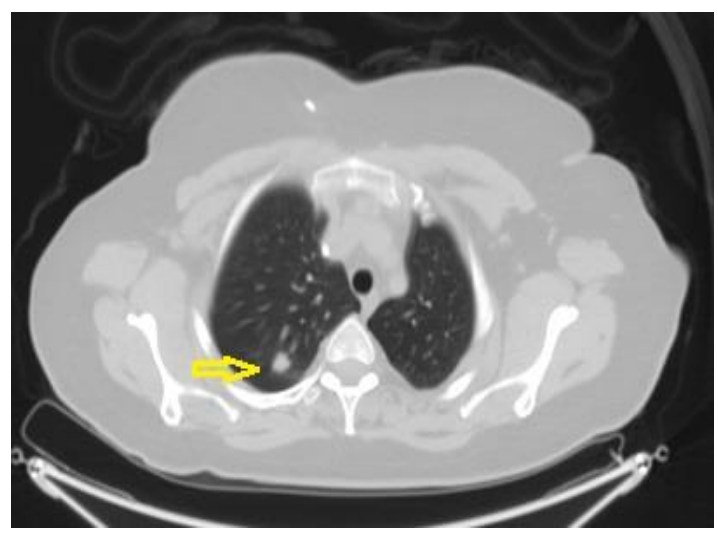

A

Figure (5): a. CT scan chest (lung window axial cuts) showed right upper lung lobe nodule (yellow arrow). b. PET/CT scan (axial images) showed FGD-avid right upper lung lobe (yellow arrow) with SUVmax $=4.9$, consistent with pulmonary metastatic deposits.

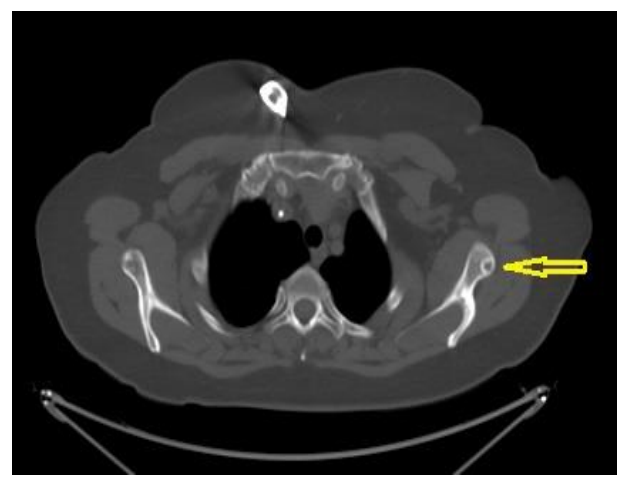

A
Whole body FDG PET/CT was done with multiple metastatic deposits denoted at dorsal vertebra, lumbar vertebra, both scapula, both iliac bones, ribs and right upper lung lobe.

$>$ The following images shows the sites of metastatic deposits (Figure 5 \& 6).

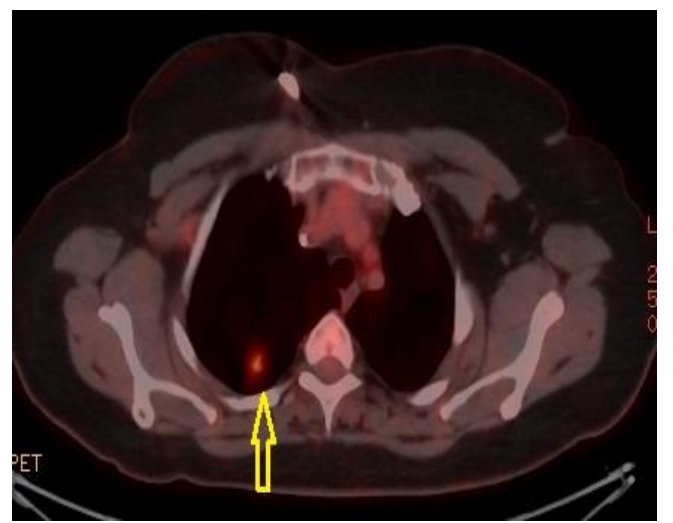

\section{B}




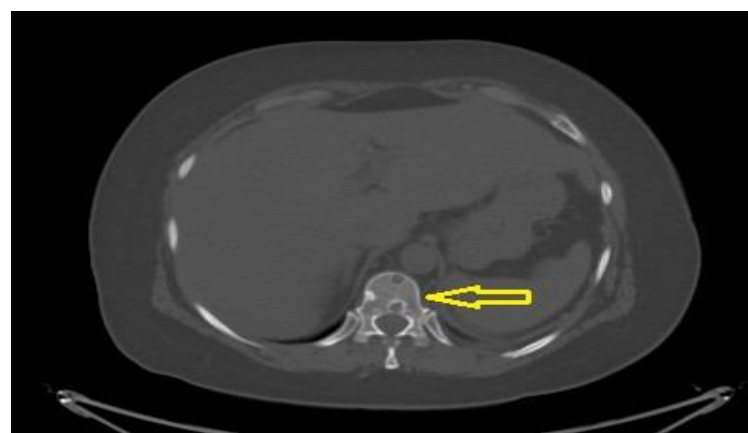

C

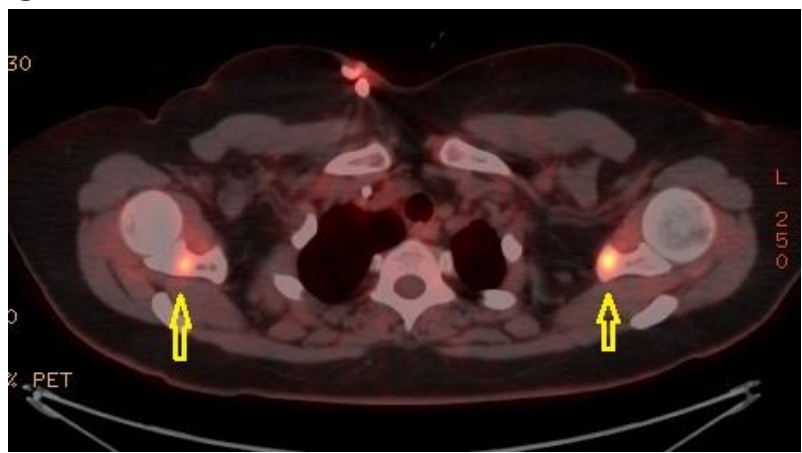

$\mathbf{E}$

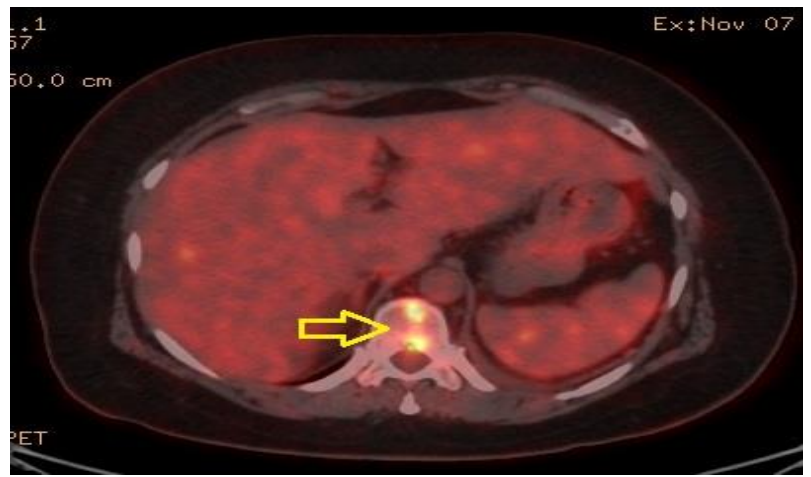

G

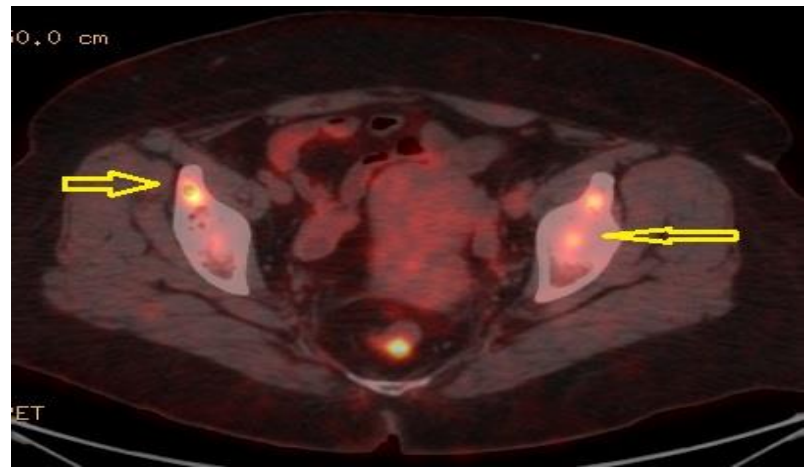

I

Figure (6): a., b., c. \& d. CT scan chest, abdomen and pelvis (bone window axial cuts) showed multiple scattered variable size intra-osseous mixed sclerotic and osteolytic deposits seen at dorsal and lumbar vertebra, scapula, right ribs and both iliac bones (yellow arrows). e., f., g., h., i. \& j. PET/CT scan (axial, coronal and sagittal images) showed multiple scattered FGD-avid intra-osseous deposits seen at dorsal vertebra, lumbar vertebra, both iliac bones, both scapula and right ribs (yellow arrows) with SUVmax $=8.1-10.5$, consistent with multiple metastatic bony deposits. 


\section{Case No. 3}

$>$ A 42 years old female patient history of left breast cancer since July 2016 underwent modified radical mastectomy at October 2017, followed by multiple cycles of chemotherapy and radiotherapy.

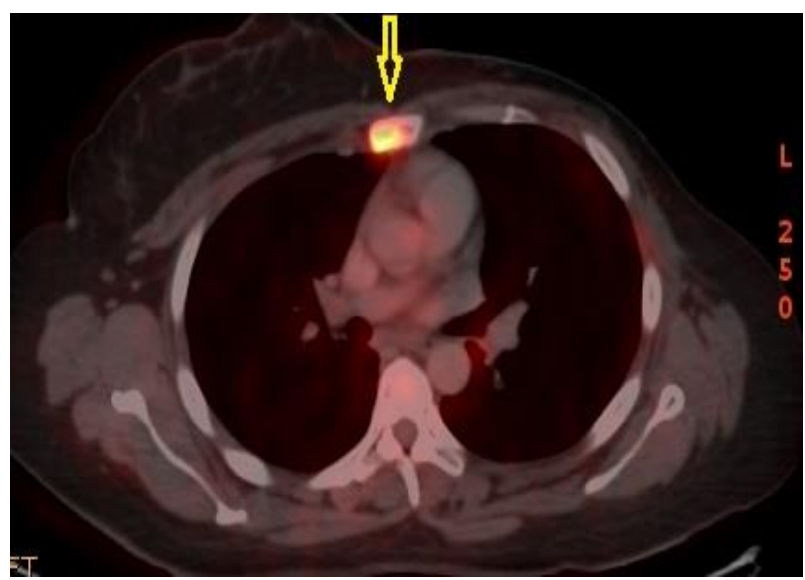

A

Figure (7): a. PET/CT (axial images) showed FDG-avid intra-osseous bony lesion is seen at the sternum (yellow arrow) with SUVmax $=5.4$ consistent with metastatic bony deposits. b. CT scan of the chest (bone window axial cuts) showed an ill-defined intra-osseous osteolytic bony lesion is seen at the sternum (yellow arrow).

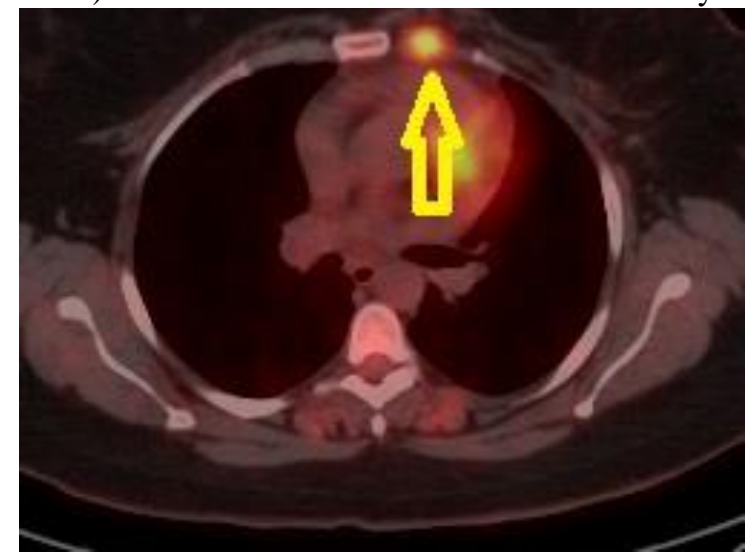

A

Figure (8): a. PET/CT scan axial cuts showed FGD-avid enlarged left sub-pectoral lymph nodes lymph nodes with SUVmax $=4.7$ consistent with metabolically active loco-regional metastatic deposits. b. CT Scan of the chest (soft tissue window axial cuts) showed enlarged left sub-pectoral lymph nodes measure 3 x $2 \mathrm{~cm}$ (yellow arrows). 


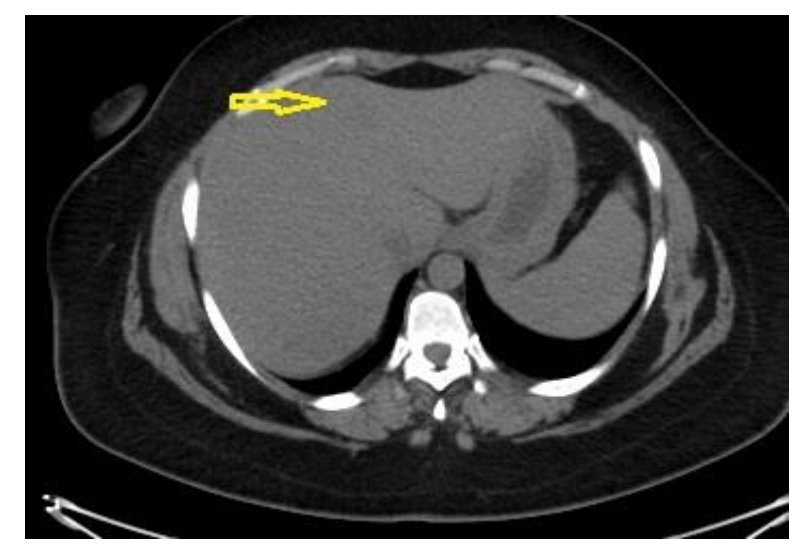

A

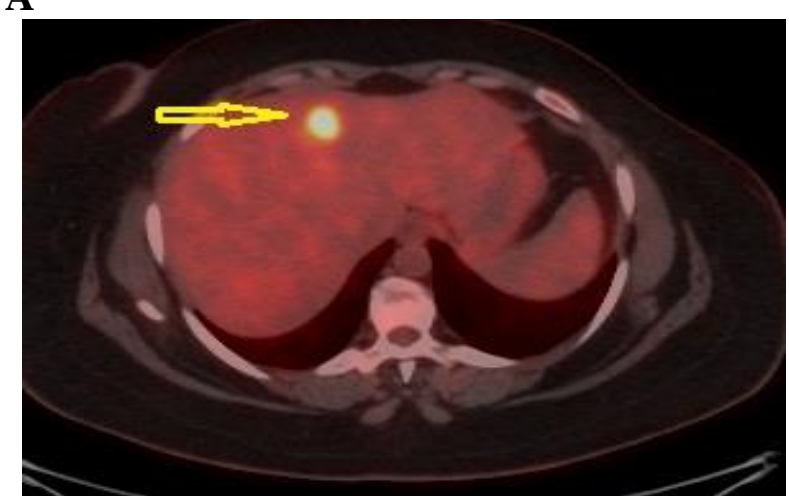

C

Figure (9): a. \& b. CT scan of the abdomen (soft tissue windor slightly hypodense lesions, the largest measure $2.5 \times 2 \mathrm{~cm}$ (yellow arrows). c. \& d. PET/CT scan (axial images) showed FGD-avid both hepatic lobes lesions (yellow arrow) with SUVmax $=11.2$ consistent denoting hepatic metastatic deposits.

\section{Case No.4}

A 70 years old female patient history of right breast cancer since November 2016 underwent lumpectomy at April 2017, followed by multiple cycles of chemotherapy and radiotherapy.
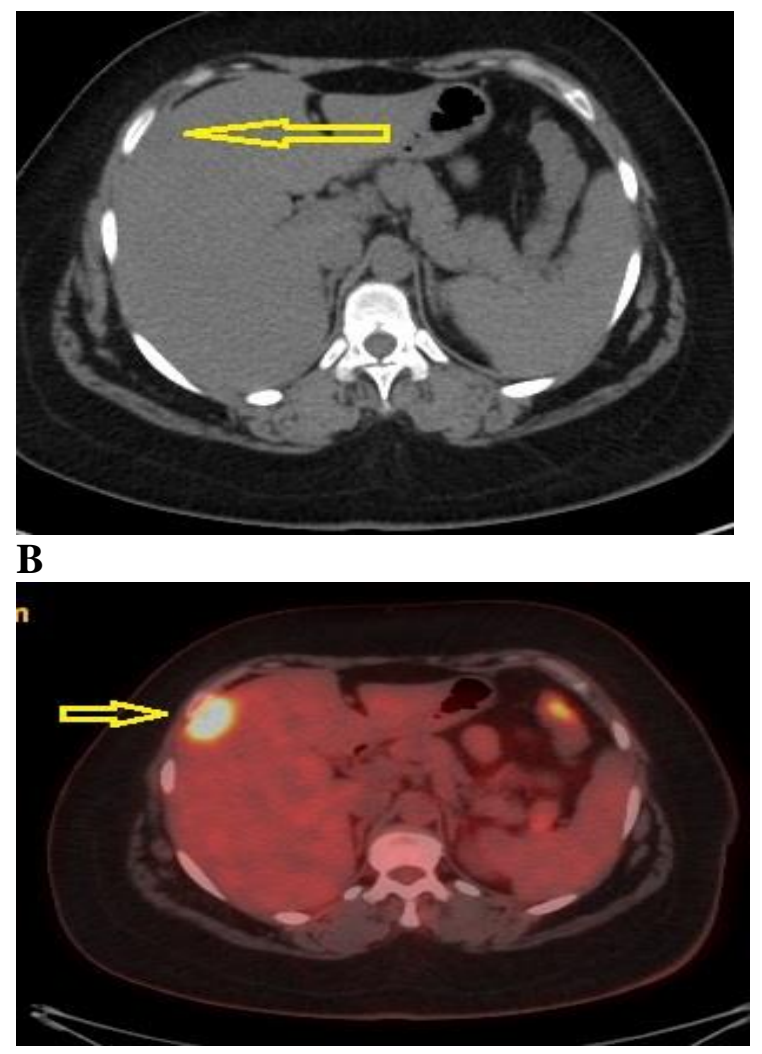

D 


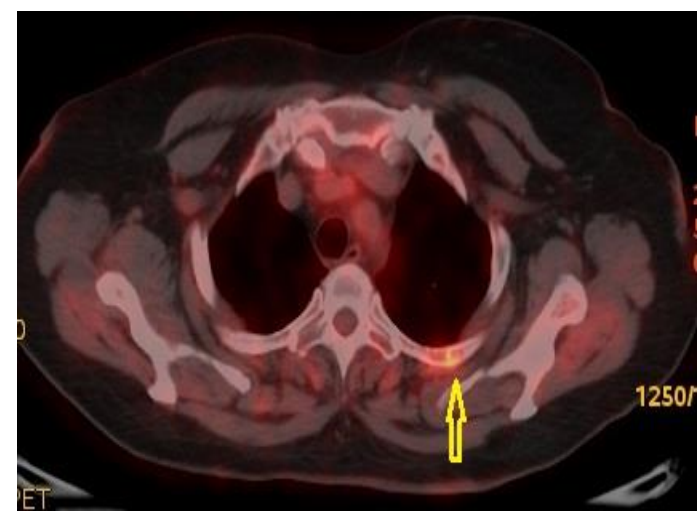

A

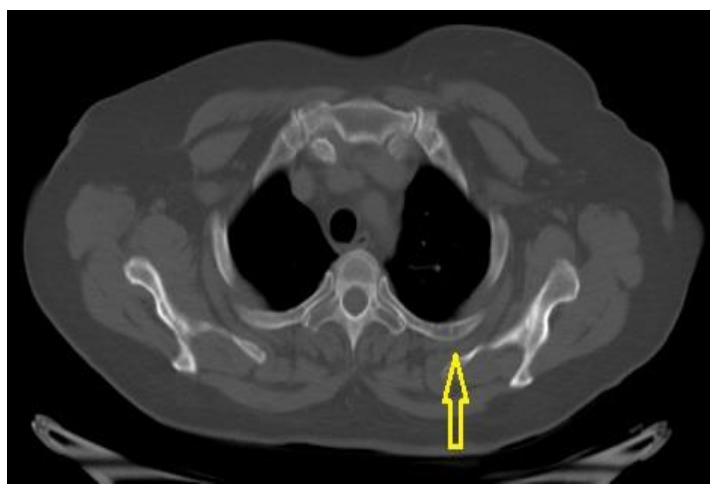

B

Figure (10): a. PET/CT (axial images) showed FDG-avid intra-osseous bony lesion is seen at the left $3^{\text {rd }}$ rib (yellow arrow) with SUVmax $=6.7$ consistent with metastatic bony deposits. b. CT scan of the chest (bone window axial cuts) showed an ill-defined intra-osseous osteolytic bony lesion is seen at the left $3^{\text {rd }}$ rib (yellow arrow).

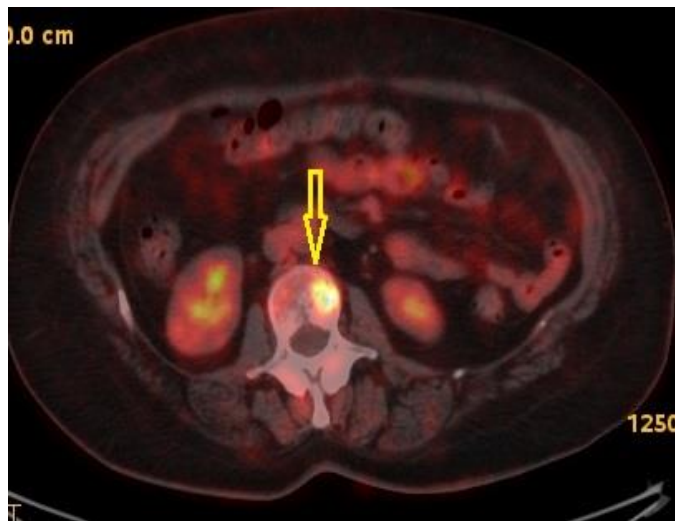

A

Figure (11): a. PET/CT (axial images) showed FDG-avid intra-osseous bony lesion is seen at the $2^{\text {nd }}$ lumbar vertebra (yellow arrow) with SUVmax $=14$ consistent with metastatic bony deposits. b. CT scan of the abdomen (bone window axial cuts) showed an ill-defined intra-osseous sclerotic bony lesion is seen at the $2^{\text {nd }}$ lumbar vertebra (yellow arrow).

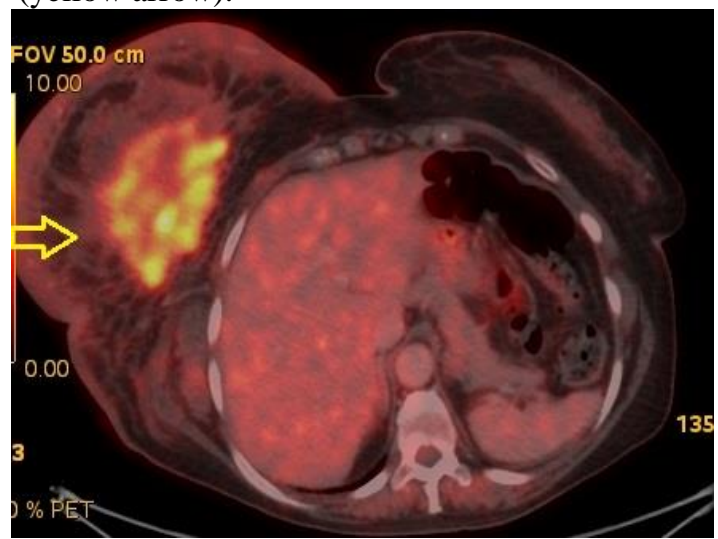

A

Figure (12): a. PET/CT scan axial cuts showed FGD-avid parenchymal lesions seen at the right breast with SUVmax $=8$ consistent with metabolically active neoplastic tissues with loco-regional metastatic deposits. b. CT Scan of the chest (soft tissue window axial cuts) showed diffuse increased skin and subcutaneous tissue thickness of the right breast with diffuse parenchymal heterongenecity as well as ill-defined soft tissue density masse lesion measure 12.8 x $5.9 \mathrm{~cm}$ (yellow arrows). 


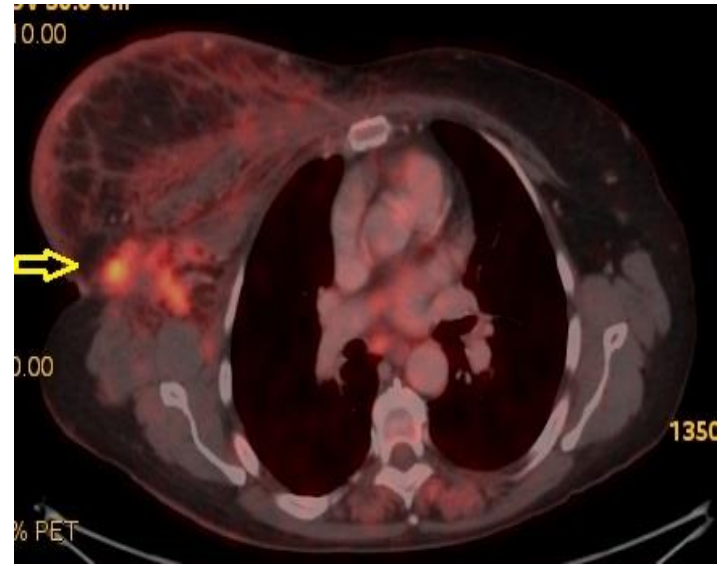

A

Figure (13): a. PET/CT scan axial cuts showed FGD-avid enlarged right axillary lymph nodes with SUVmax $=5.3$ consistent with metabolically active loco-regional metastatic deposits. b. CT Scan of the chest (soft tissue window axial cuts) showed enlarged right axillary lymph nodes measure 3 x $5 \mathrm{~cm}$ (yellow arrows).

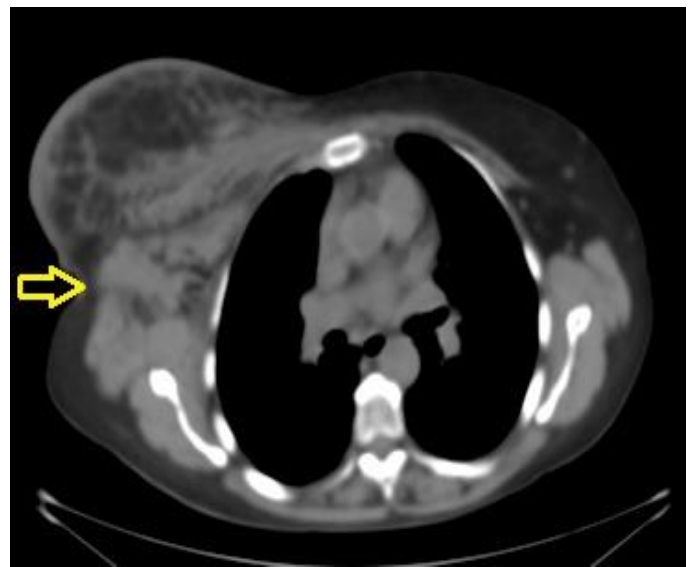

B

\section{Conclusion:}

$\mathrm{PET} / \mathrm{CT}$ is whole body single imaging which can detect metastatic spread in breast cancer as well as monitoring the therapy response in relation to different molecular subtypes.

\section{F-2-fluoro-2-deoxy-D-glucose}

(FDG) positron emission tomography (PET) is able to supply functional information for wholebody tumor staging with higher sensitivity, specificity and accuracy as compared to $\mathrm{CT}$ in detection of metastatic spread and follow up after treatment.

PET/CT was requested for initial staging of the included cases then evaluated qualitatively and semis quantitatively with calculation of SUV max.

\section{References:}

1. Jemal A , Bray F , Center MM , Ferlay J , Ward E , Foreman D. Survillance researches , American Scocity breast cancer statistics 2011 Mar-Apr;61 (2):69-90.

2. Lacey J.V. , Jr. , Kreimer A.R. , Buys S.S. , Marcus P.M. , Chang S.C. et al. Breast cancer epidemiology according to recognized breast cancer risk factors and screening trial cohort. BMC Cancer. 2009;9:84.

3. Iagaru A , Masamed R, Keesara S , Conti P.S. Breast MRI and ${ }^{18} \mathrm{~F}$ FDG PET/CT in the management of breast cancer. Journal of Nuclear Medicine 2007;21:33-38.

4. Zahang X., Wu F., Han P. The role of 18FFDG PET/CT in the diagnosis of breast cancer and lymph nodes metastases and micro- 
metastases may be limited. Hell. Journal of Nuclear Medicine. 2014;17:177-183.

5. Kobayashi katsuhiro, Bhargava Peeyush , Raja Shanker. What the radiologists need to know about PET/CT. Radiographics 2012;32:1483-1501.

6. Brunetti , JC. PET and FDG PET-CT imaging of breast cancer. Applied radiology (2009);38(9).

7. Esserman L. Integration of imaging in the management of breast cancer. Journal of Clinical oncology. 2005;23:1601-1602.

8.Surasi D, Bhambhvani P, Baldwin J. (18)F-FDG PET and PET/CT patient preparation: a review of the literature. Journal of Nuclear Medicine Technology . 2014; 42(1):5-13.

9.Radiological Society of North America. FDGPET/CT UPICT V1.0.http://www.rsna.org/uploadedFiles/RSNA/ Content/Science and Education /QIBA/UPICT FDGPET Protocol ver08July2014.pdf. Accessed June 30, 2015.

10. Stopeck T. Breast cancer. Medscape (serial online). Available at <http://Medscape.com/view article/1276001; 2014 [accessed 7.04.14].

11. Cappelletti V, Iorio E, Miodini P, Silvestri M, Daidone MG. Distant Markers. 2017;2017:7687851. doi: 10.1155/2017/7687851. Epub 2017 Dec 24.

12. Aukema TS, Straver ME, Peeters MJ. Detection of extra-axillary lymph node involvement with FDG PET/CT in patients with stage II-III breast cancer. European Cancer journal 2010;46:3205-10.

13. Graham LJ, Matthew P, Shupe PM, Shumway MN. Current approaches and challenges in monitoring treatment responses in breast cancer. J Cancer 2014;5(1):58-86.

14. Higuchi T, Nishimukai A, Ozawa H, Fujimoto Y, Yanai A, Miyagawa Y. et al. 32016 Dec;30:5-12. doi: 10.1016/j.breast.2016.08.003. Epub 2016 Aug 29.

15. Niikura N, Costelloe CM, Madewell JE. FDGPET/CT compared with conventional imaging in the detection of distant metastases of primary breast cancer. Oncologist 2011;16(8): 1111-9.

16. Hahn S, Heusner T, Ku“ mmel S. Comparison of FDG-PET/ CT and bone scintigraphy for detection of bone metastases in breast cancer. 2011;52:1009-14.

17. Eubank WB, Mankoff DA, Takasugi J. 18fluorodeoxyglucosepositron emission tomography to detect mediastinal or internal mammary metastases in breast cancer. Journal of Clinical Oncology 2001;19(15):3516-23.

18. Garami Z, Hascsi Z, Varga J. The value of 18FDG PET/ CT in early-stage breast cancer compared to traditional diagnostic modalities with an emphasis on changes in disease stage designation and treatment plan. European Journal of Surgical Oncology 2012;38:31-7.

19. Kennecke H, Yerushalmi R, Woods R. Metastatic behavior of breast cancer subtypes. Journal of Clinical Oncology 2010;28:3271-7.

To cite this article: Medhat M. Reffat, Haney H. Lotfy, Ahmed L. Salem. Role of 18FDGPET/CT in evaluation of breast cancer metastasis. BMFJ 2022; 39 (Radiology):133-148. DOI: $10.21608 / \mathrm{bmfj} .2021 .85158 .1442$ 